\title{
THE USNO ASTROMETRIC INTERFEROMETER
}

\author{
DONALD J. HUTTER \\ United States Naval Observatory \\ USNO/NRL Optical Interferometer Project, AD5 \\ 3450 Massachusetts Avenue, NW \\ Washington, DC 20392-5420
}

\begin{abstract}
The U.S. Naval Observatory Astrometric Interferometer (USNOAI) will be the dedicated astrometric sub-array of the new Navy Prototype Optical Interferometer (NPOI) at Lowell Observatory, which is being built in collaboration with the Naval Research Laboratory. The USNOAI will be in operation on Anderson Mesa, near Flagstaff, Arizona, in late 1993. The instrument will incorporate four siderostats which will be located in a Y-shaped configuration, and will feature state-of-the-art delay lines, a real-time zeroth-order fringe tracking algorithm, a full-array laser metrology system to monitor baseline motion, and a limiting magnitude of 10 . The instrument will be capable of simultaneous observations on six baselines, and will produce star positions accurate to a few mas. With it, we will be able to maintain the HIPPARCOS reference frame with matching accuracy over the next few decades.
\end{abstract}

\section{Introduction}

The USNOAI is being built using the experience gained from the Mark III Interferometer on Mt. Wilson, CA, which has demonstrated "proof of concept" of wide-angle astrometry by a long baseline optical interferometer. The Mark III is a broad-band phase-tracking optical interferometer, which became operational in late 1986 (Shao et al. 1988). The Mark III is capable of single baseline measurements on either a north-south or east-south baseline, each of about 12 meters in length. The Mark III incorporates evacuated delay lines with automated high speed servo systems allowing the detection and tracking of whitelight fringes in real time. A method using three different observing wavebands is employed which corrects for delay variations introduced by turbulent spectral-index fluctuations of the atmosphere.

Measurements of stellar positions by the Mark III in a selected subset of the FK5 catalog, made at several epochs over a five-year period, have formal uncertainties of 5 to 10 mas (Shao et al. 1990; Hummel 1993). There are, however, small systematic errors present in these solutions, and they manifest themselves in two ways. Firstly, the solutions depend strongly on the choice of FK5 stars used in the analysis; the systematic errors of the initial FK5 positions are propagated to the solution for the baseline and ultimately to the corrections for the FK5 positions. Secondly, there are mechanical and thermal motions within the instrument. These motions produce systematic errors which are noticeable when the same set of FK5 stars are observed on more than one night. In order to remove the systematic errors due to the choice of FK5 stars, four siderostats have been incorporated into the final

${ }^{1}$ The Naval Research Laboratory is constructing the imaging sub-array of the NPOI, the "Big Optical Array" or BOA (Simon 1993). 
USNOAI design. Thus there will be six baselines, three of which are geometrically independent. An instrument with at least three independent baselines allows the total separation of the baseline components and star position corrections in the astrometric solutions. To further reduce systematic errors, an extensive baseline metrology system is employed to fully monitor the motions of the baselines in three dimensions with respect to a reference system fixed to the earth's crust. In addition to these improvements, the increased sensitivity of the USNOAI will allow the measurement of the positions of some of the brighter stellar radio sources, whose positions have been tied to the radio reference frame defined by extragalactic sources.

The combination of these improvements will permit the USNOAI to produce highly accurate catalogs of star positions on a nearly inertial frame. The initial goal of the USNOAI will be to establish, by mid 1996, a catalog of the order of one thousand stars with statistical and systematic errors in the range of 1 to 3 mas. This catalog will be more than an order-of-magnitude more precise than the FK5. The stars in the new catalog, if their positions are measured over a period of ten years, will have proper motions known to the order of 1 mas.

Thus the USNOAI, with a capability of milliarcsecond astrometry and a planned operational lifetime of several decades, will be capable of maintaining the HIPPARCOS reference frame through repeated observations, yielding improved proper motions, of thousands of the brighter HIPPARCOS stars.

\section{Description of the Instrument}

The layout of the USNOAI is shown in Figure 1. Vacuum pipes extend out each arm of the array to the siderostat stations. The lines leading from the array center carry the starlight beams to the optical laboratory. This structure will contain the delay lines, beam combiners, fringe detection, and narrow angle tracking systems. All interferometer systems will be remotely controlled from a separate operations facility.

\subsection{SIDEROSTATS}

The siderostats and associated systems are shown in Figure 2. The siderostats will be housed in roll-off-roof enclosures, which will be climate controlled during daylight hours. The siderostats are modified versions of those to be used with the BOA. They consist of permanently mounted 2-axis alt-az mountings which each support a flat mirror of $50 \mathrm{~cm}$ clear aperture. The siderostats are driven in each axis by a novel drive system consisting of in-line dual harmonic drive gear reduction units driven by stepper motors. A wide field-of-view star acquisition capability is also incorporated via a CCD camera located near each siderostat. A 2-axis gimble mounted flat mirror, with piezo-electric actuators, will be located in the stellar light path near each siderostat to provide the small angle, high speed tip-tilt correction necessary to compensate for random image motion due to atmosphere turbulence. Provision will be made within the siderostat enclosures for the future addition of a $35 \mathrm{~cm}$ aperture afocal beam compressor next to each siderostat. The beam compressors would permit a significant increase in the limiting sensitivity of the 


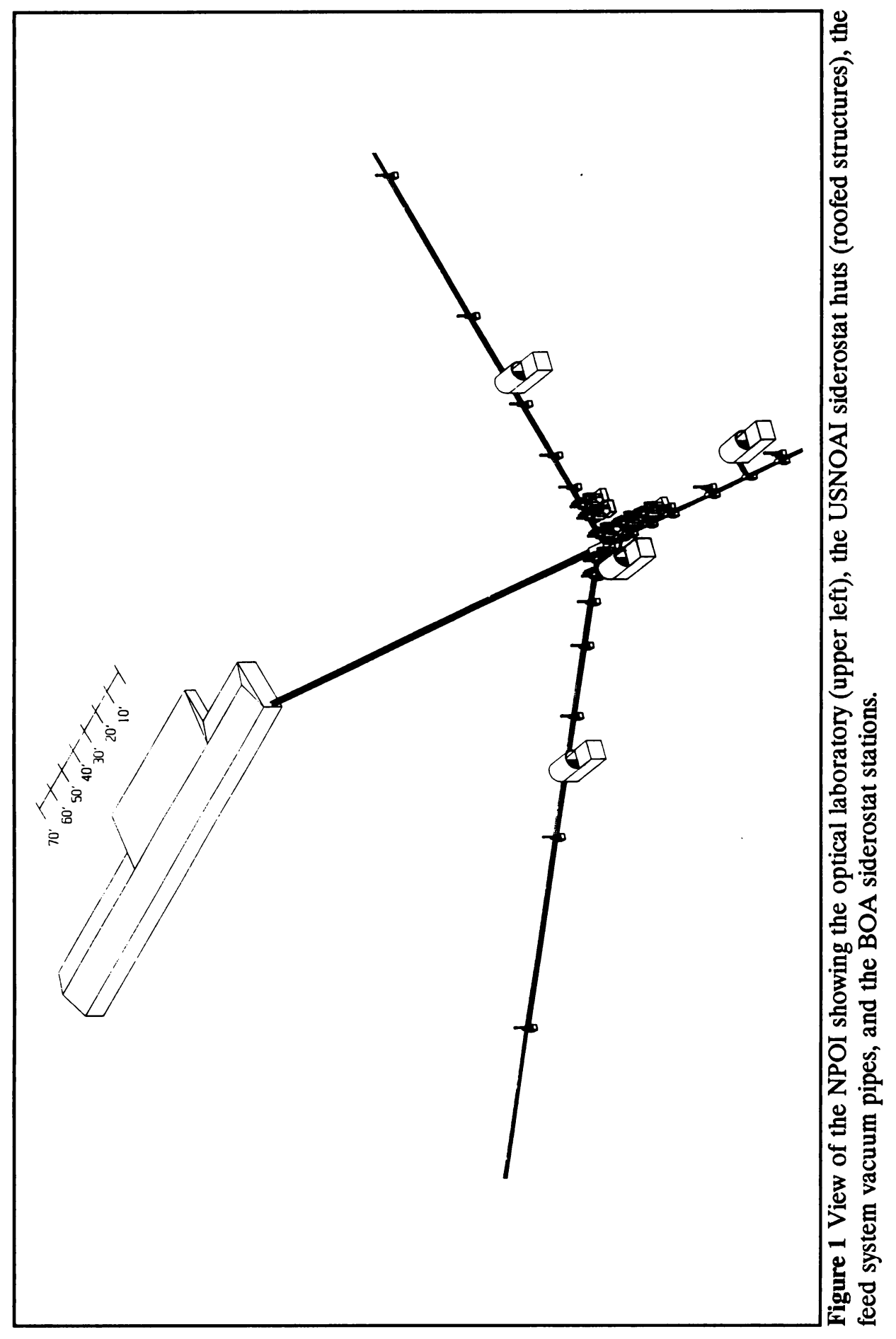




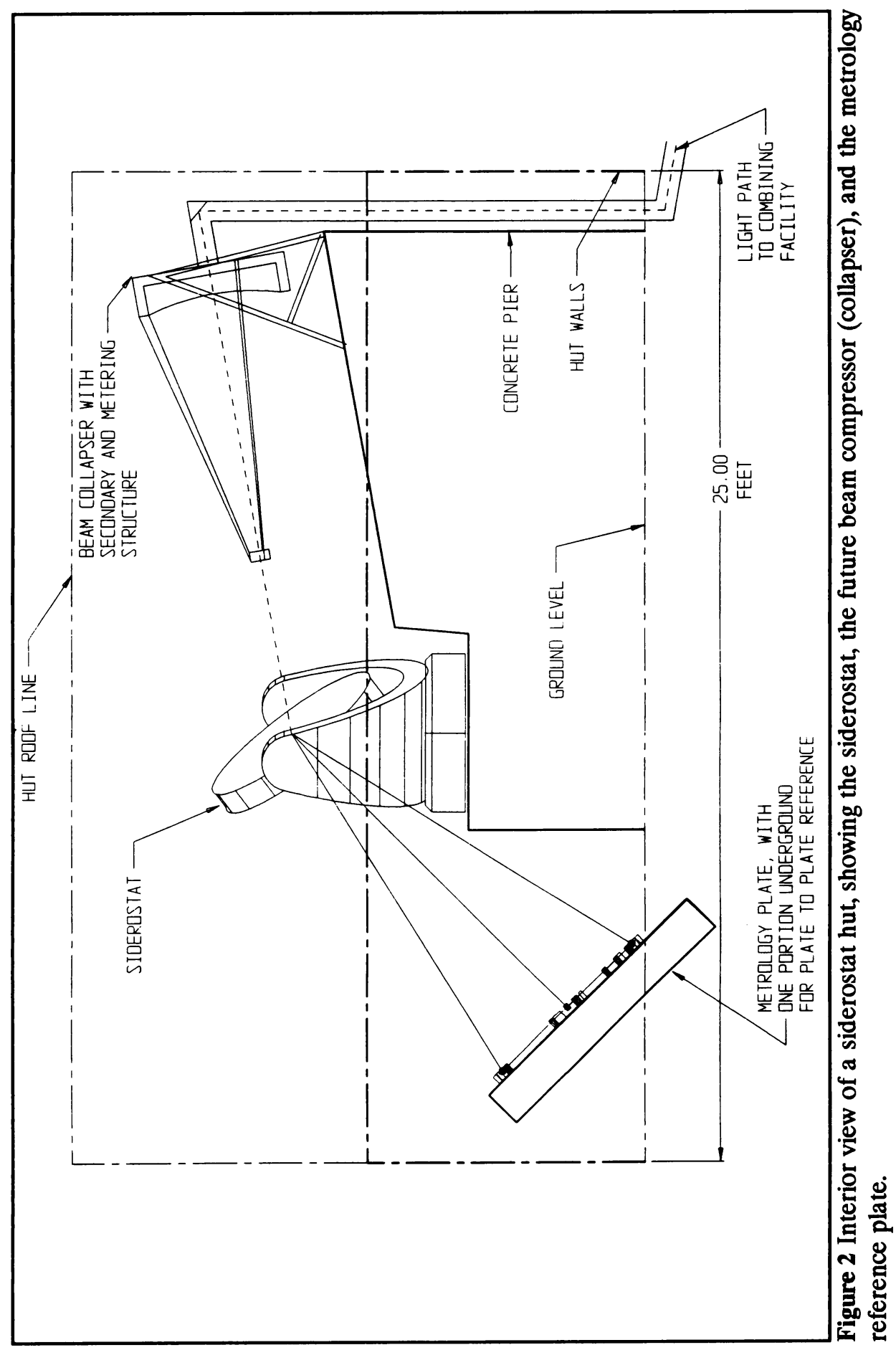


USNOAI through observations in the infrared or through the use of multi- $r_{0}$ groupdelay techniques at visible wavelengths, both of which could effectively exploit the full aperture of each siderostat.

\subsection{METROLOGY SYSTEM}

In order that the USNOAI achieve its design goal of mas astrometry, a baseline laser metrology system must be employed which can measure the three-dimensional motions of the baselines to an accuracy of approximately $0.1 \mu \mathrm{m}$ (Elias and Hutter 1993). This system consists of a number of laser interferometers tied to each of four temperature stabilized super-Invar reference surfaces, one adjacent to each siderostat (Figure 2). Five laser interferometers measure the position of a retroreflector at the intersection of the rotation axes of each siderostat, relative to the adjacent reference plate (Figure 3). The rotation and tilt of the reference plates will, in turn, be measured by two other metrology subsystems. In the first case, laser interferometers will monitor changes in the distances of several points on each plate from retroreflectors embedded in a deep subsurface layer. The second subsystem consists of interferometers along lines-of-sight between the reference plates at the various siderostats to detect motions of the plates in the horizontal plane (Figure 4). Together, these various subsystems contain 46 laser interferometers which together will allow a continuous measurement of the time evolution of the baselines of the USNOAI with respect to an earth-fixed reference system with submicron error.

Changes in the delay zero-point (or constant term) on each interferometer baseline will also be continuously monitored by means of infrared laser metrology beams which will traverse the same optical path as the stellar beams. The value of the delay-constant will be periodically measured by means of fringe tracking on an internal white-light source, in a manner similar to the employed on the Mark III.

\subsection{DELAY LINES}

The USNOAI shares with the BOA six optical delay lines, each providing up to 35 $m$ of optical delay. The prototype of these delay lines was developed under contract at the Jet Propulsion Laboratory. These delay lines are similar to those in use with the Mark III Interferometer, but have smoother support and drive mechanisms, in order to operate successfully at the higher speeds and greater lengths required by the USNOAI. The prototype of these delay lines is capable of active fringe tracking at typical sidereal fringe motion rates with an RMS smoothness of $10 \mathrm{~nm}$. The position of the delay line retroreflector will be measured using infrared laser metrology. As in the Mark III, the delay lines will be entirely in vacuum to allow the widest possible observational bandwidth.

\subsection{BEAM COMBINER}

Unlike the Mark III, which was limited to tracking stellar fringe on only a single baseline at a time, the USNOAI will be capable of simultaneous observations on all of its six available baselines. This is made possible by the larger number of delay lines employed, and by significantly improved beam combination and fringe detection systems. 


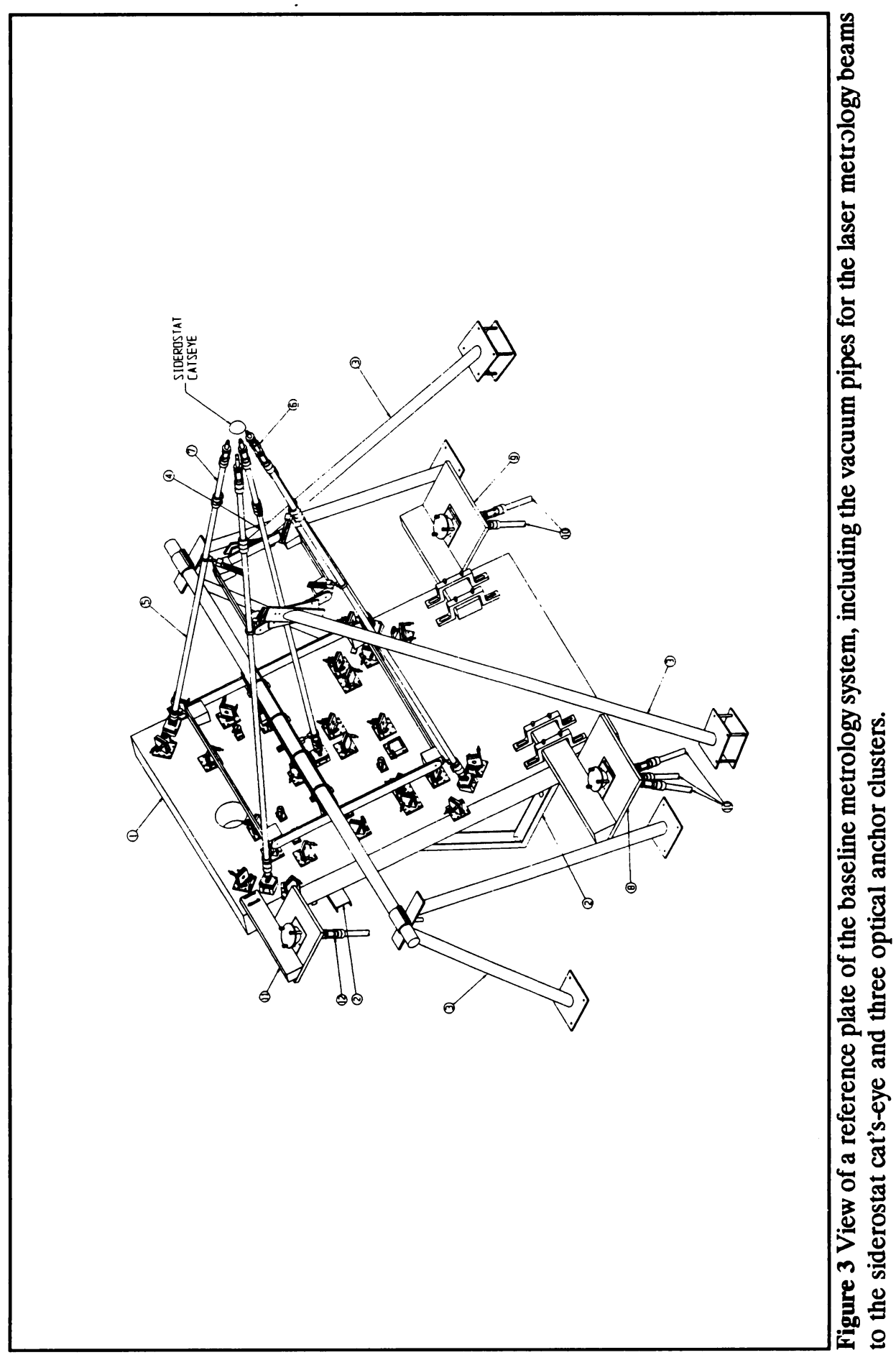




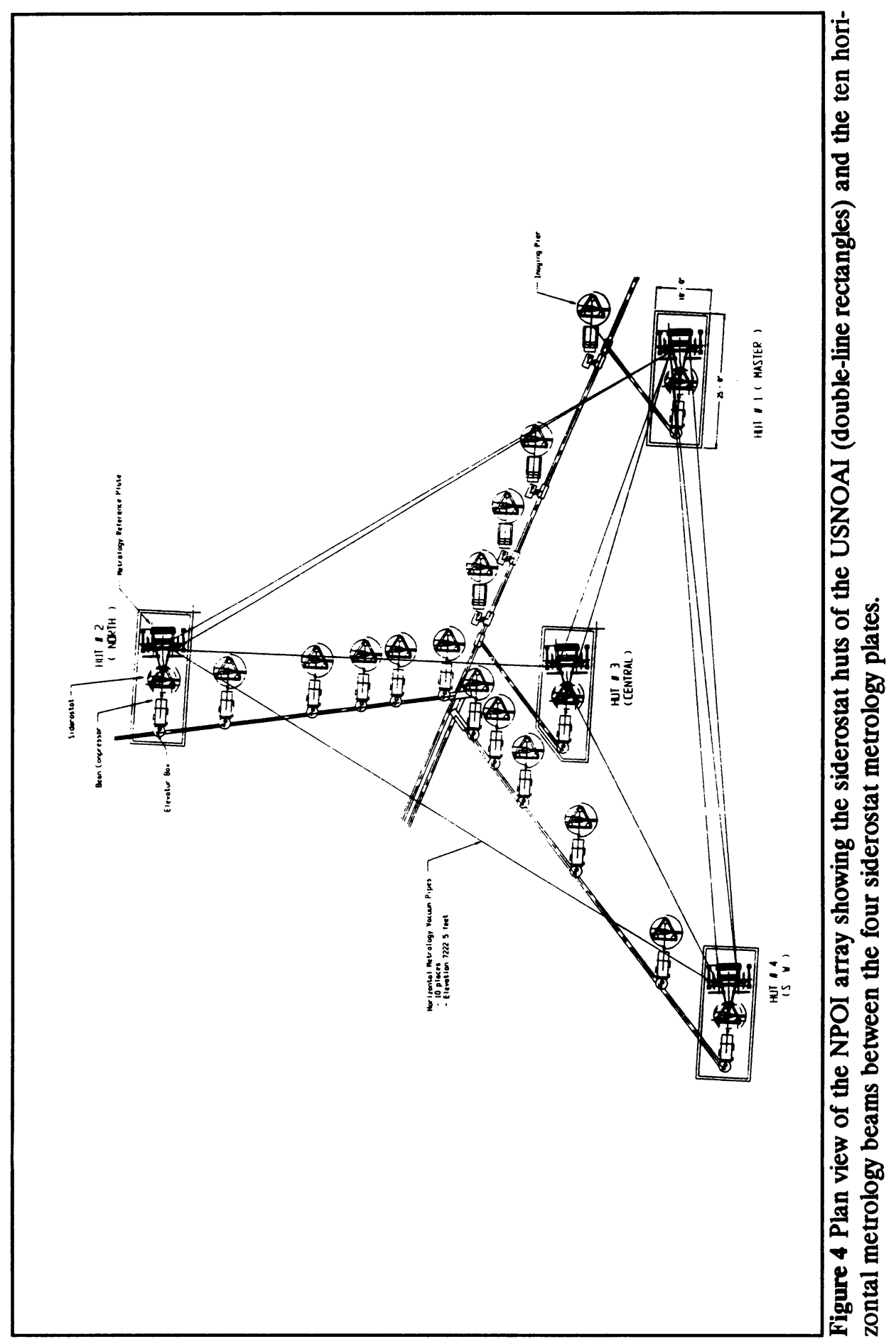


The beam combining optics of the USNOAI (Mozurkewich 1993) will combine the beams from all four siderostats in a pair-wise fashion, determining the fringe parameters on each baseline separately. A prototype three-beam version of this system has been built and currently has system visibility amplitudes of $90 \%$. This type of beam combiner design allows angle tracking using combined images and should have good stability because common optics are used for both the angle and fringe sensors.

\subsection{FRINGE DETECTION}

The fringe sensing system (Mozurkewich 1993) disperses the fringe in both wavelength and delay. To implement this sensor, a high quantum efficiency, photon counting array using single avalanche photodiodes and relatively inexpensive optics was constructed. Although the beam combiner and fringe sensor systems were originally conceived for imaging work with the BOA, they offer significant advantages for astrometric observations over the techniques employed on the Mark III. In addition to the multi-way beam combiner allowing simultaneous observations on all of the available baselines, the dispersed fringe detection technique allows unambiguous central fringe identification, and better correction for atmospherically induced delay fluctuations.

\section{Milestones}

Construction of the USNOAI is proceeding rapidly. Prototypes of most of the major subsystems have been completed. The siderostat and delay line prototypes have been tested and the fabrication and assembly of additional units is well advanced. The construction of the electronics and software for the various USNOAI subsystems (Buchanan 1993) continues in parallel with that of the mechanical and optical systems.

Construction at the interferometer site on Anderson Mesa began in the late summer of 1992 (White 1993). Access roads and the interferometer control building are now nearly complete. The construction of the optical laboratory is expected to begin in March 1993. All construction at the site should be completed by late summer, and all of the interferometer components should be installed by early autumn. Limited USNOAI operations are planned to begin by November 1993, and routine astrometric observations with four siderostats are expected by mid-1994.

\section{Conclusion}

The USNOAI, with a capability of milliarcsecond astrometry and a planned operational lifetime of several decades, will be capable of maintaining the HIPPARCOS reference frame through repeated observations, yielding improved proper motions, of thousands of the brighter HIPPARCOS stars. 


\section{References}

Buchanan, K.: 1993, Real Time Control of a Long Baseline Optical Interferometer, in The Proceedings of IAU Symposium No. 158

Elias, N.M., and Hutter, D.J.: 1993, The Baseline Metrology System of the USNO Astrometric Interferometer, in The Proceedings of LAU Symposium No. 158

Hummel, C.A: 1993, Five Years of Stellar Position Measurements with the MkIII Optical Interferometer, in The Proceedings of LAU Symposium No. 158

Mozurkewich, D.: 1993, Design and Construction of a Beam Combiner and Related Hardware, in The Proceedings of LAU Sympasium No. 158

Shao, M., Colavita, M.M., Hines, B.E., Staelin, D.H., Hutter, D.J., Johnston, K.J., Mozurkewich, D., Simon, R.S., Hershey, J.L, Hughes, J.A., and Kaplan, G.H.: 1988, Astron. Astrophys. 193, 357

Shao, M., Colavita, M.M., Hines, B.E., Hershey, J.L, Hughes, J.A, Hutter, D.J., Kaplan, G.H., Johnston, K.J., Mozurkewich, D., Simon, R.S., and Pan, XP.: 1990, $A J$ 100, 1701

Simon, R.S.: 1993, The Big Optical Array, in The Proceedings of LAU Sympasium No. 158

White, N.M.: 1993, Site Development of the Navy Prototype Optical Interferometer (NPOI) at the Lowell Observatory, in The Proceedings of IAU Symposium No. 158 